PROCEEDINGS OF THE

AMERICAN MATHEMATICAL SOCIETY

Volume 140, Number 3, March 2012, Pages 941-949

S 0002-9939(2011)10978-X

Article electronically published on July 15, 2011

\title{
WEIGHTED PALEY-WIENER SPACES SHARING A MAJORANT-WEIGHT
}

\author{
PHILIPPE POULIN
}

(Communicated by Richard Rochberg)

\begin{abstract}
We point out a need to slightly modify the statement of Theorem 2 in Yurii Lyubarskii and Kristian Seip's work Weighted Paley-Wiener spaces. This last theorem lists all weighted Paley-Wiener spaces (in reduced form) sharing a prescribed majorant-weight. Attention is called to a part of its proof that requires an additional argument. Such an argument, based on a new characterization of Beurling's lower uniform density, is then presented.
\end{abstract}

\section{INTRODUCTION}

In the explorative paper [1, Lyubarskii and Seip introduced a family of de Branges spaces subject to a natural axiom: in these spaces, the norm of a function is comparable to its $L^{2}$-norm against $M(x)^{-2} \mathrm{~d} x$, where $M(x)$ denotes the norm of the reproducing kernel at $x \in \mathbb{R}$ (see Section 2). In such circumstances, $M(x)$ is said to be a majorant-weight, while the de Branges space is said to be a weighted Paley-Wiener space.

From a deep study of the Hermite-Biehler function associated with weighted Paley-Wiener spaces (Theorem 1 in [1]), they showed that the majorant-weight of a weighted Paley-Wiener space is always comparable to a function of the form $\mathrm{e}^{g(x)} \mathrm{e}^{\omega_{m}(x)}$, where $g(z)$ is real-entire (that is, real on the real line and entire), $m(x)$ is comparable to a constant, and $\omega_{m}(z)$ is the potential of $m(x) \mathrm{d} x$ (see Section 2).

Furthermore, they proved that any weighted Paley-Wiener space is of the form $\mathrm{e}^{g} P W(m)$ for such a $g$ and an $m$, where

$$
P W(m)=\left\{f \text { entire; }\left\|f \mathrm{e}^{-\omega_{m}}\right\|_{2}<\infty,|f(z)| \mathrm{e}^{-\omega_{m}(z)} \leq C_{\varepsilon} \mathrm{e}^{\varepsilon|z|}\right\} .
$$

They then aimed to list all weighted Paley-Wiener spaces of the form $P W(\cdot)$ whose majorant-weight is comparable to a prescribed $\mathrm{e}^{\omega_{m}(x)}$. They obtained such a list (Theorem 2 in [1]), which consists exactly of the following spaces,

$$
P W_{-b}(m)=\left\{f \text { entire } ;\left\|f \mathrm{e}^{-\omega_{m}}\right\|_{2}<\infty,|f(z)| \mathrm{e}^{-\omega_{m}(z)+\pi b|\Im z|} \leq C_{\varepsilon} \mathrm{e}^{\varepsilon|z|}\right\},
$$

where $b$ is any real number inferior to the lower uniform density of $m$. This last is defined as

$$
D_{m}=\lim _{R \rightarrow \infty} \inf _{x \in \mathbb{R}} \frac{1}{2 R} \int_{-R}^{R} m(x+t) \mathrm{d} t .
$$

Received by the editors December 16, 2010.

2010 Mathematics Subject Classification. Primary 30H99.

Key words and phrases. de Branges space, weighted Paley-Wiener space, majorant-weight.

(C)2011 American Mathematical Society 
However, as we shall discuss later, a close examination of their proof reveals its incompleteness, and new ideas seem necessary for completing their work.

The present paper aims to remedy the situation. Section 2 provides the reader with the main definitions used in the sequel. In Section 3.1, we shall question the original argument that $D_{m}$ is a majorant of $b$ and then provide our own proof, based on a new characterization of the lower uniform density. Finally, in Section 3.2, we shall show that $D_{m}$ is the least majorant of $b$.

\section{Definitions}

In the sequel, given two nonnegative functions $f$ and $g, f \lesssim g$ indicates that $f \leq C g$ for a positive constant $C$, and $f \simeq g$ indicates that $f$ is comparable to $g$ (that is, $f \lesssim g$ and $g \lesssim f$ ).

A Hilbert space $\mathcal{H}$ of entire functions is a de Branges space [2] if it satisfies the following axioms:

(1) The linear functional $\mathcal{H} \rightarrow \mathbb{C}, f \mapsto f\left(z_{0}\right)$ is bounded for all $z_{0} \in \mathbb{C}$.

(2) If $f(z) \in \mathcal{H}$, then $f^{*}(z)=\overline{f(\bar{z})}$ also belongs to $\mathcal{H}$ and has the same norm as $f(z)$.

(3) If $f(z) \in \mathcal{H}$ and $f\left(z_{0}\right)=0$, then $f(z) \frac{z-\overline{z_{0}}}{z-z_{0}}$ also belongs to $\mathcal{H}$ and has the same norm as $f(z)$.

By the first axiom, $\mathcal{H}$ admits a reproducing kernel, that is, a function $k_{w}(z)$ of the variables $w, z \in \mathbb{C}$ such that $k_{w} \in \mathcal{H}$ for all $w \in \mathbb{C}$ and

$$
\left\langle f, k_{w}\right\rangle_{\mathcal{H}}=f(w) \text { for all } f \in \mathcal{H} \text {. }
$$

The majorant of $\mathcal{H}$ at $z \in \mathbb{C}$ is then defined as

$$
M(z)=\left\|k_{z}\right\|_{\mathcal{H}}=\sup _{\|f\|_{\mathcal{H}}=1}|f(z)| .
$$

Let $M(x)$ be the restriction of $M$ to the real axis. Following Lyubarskii and Seip, we shall say that $M(x)$ is a majorant-weight if

(1) $M(x)>0$ for all $x \in \mathbb{R}$;

(2) $\|f\|_{\mathcal{H}} \simeq\|f / M\|_{2}$ for all $f \in \mathcal{H}$.

Then, the corresponding $\mathcal{H}$ is called a weighted Paley-Wiener space.

A Hermite-Biehler function $E$ is an entire function satisfying $|E(z)|>|E(\bar{z})|$ for all $z \in \mathbb{C}^{+}$. Such a function may be factorized as

$$
E(z)=C z^{m} \mathrm{e}^{h(z)} \mathrm{e}^{-\mathrm{i} \alpha z} \prod_{\gamma \in \Gamma}(1-z / \gamma) \mathrm{e}^{z \Re(1 / \gamma)},
$$

where $C \in \mathbb{C}, h(z)$ is real-entire, $\alpha \geq 0$, and $\Gamma$ is a family of nonzero elements lying in the closed lower half-plane (with possible repetitions). Conversely, given such a $C, h(z), \alpha$, and $\Gamma$, if the right-hand side in (2.1) defines an entire function, then it is in the Hermite-Biehler class (provided that $\Gamma \nsubseteq \mathbb{R}$ or $\alpha \neq 0$ ).

In the case where $E$ does not have real zeroes, its restriction to the real axis may be written

$$
E(x)=|E(x)| \mathrm{e}^{-\mathrm{i} \varphi(x)},
$$


where the phase, $\varphi(x)$, is real-analytic and well-defined (up to the addition of $2 k \pi$ ). The factorization (2.1) then implies

$$
\varphi^{\prime}(x)=\alpha+\sum_{\xi-\mathrm{i} \eta \in \Gamma} \frac{\eta}{(x-\xi)^{2}+\eta^{2}} .
$$

From an arbitrary Hermite-Biehler function $E$, one may build a prototypical example of a de Branges space, namely

$$
\mathcal{H}(E)=\left\{f \text { entire } ;\|f / E\|_{2}<\infty,\left|f^{\sharp}(z) / E(z)\right| \leq C_{\varepsilon} \mathrm{e}^{\varepsilon|z|} \text { for } \Im z \geq 0\right\},
$$

for $f^{\sharp}$ running over $\left\{f, f^{*}\right\}$. It is equipped with the norm $\|f\|_{\mathcal{H}(E)}=\|f / E\|_{2}$. In fact, a theorem of de Branges (Theorem 23 in [2]) shows that every de Branges space is isometrically equal to a space of the form $\mathcal{H}(E)$, where $E$ is not unique in general.

The reproducing kernel in $\mathcal{H}(E)$ is given by

$$
k_{w}(z)=\frac{E^{*}(z) \overline{E^{*}(w)}-E(z) \overline{E(w)}}{2 \pi \mathrm{i}(z-\bar{w})} .
$$

In particular, if $E$ does not have real zeroes,

$$
M(x)=\sqrt{k_{x}(x)}=\frac{1}{\sqrt{\pi}} \sqrt{\varphi^{\prime}(x)}|E(x)|
$$

for all $x \in \mathbb{R}$.

Example 2.1. Let $\varphi$ be the phase of a Hermite-Biehler function $E$ without real zero. If $\varphi^{\prime}(x) \simeq 1$, then $\mathcal{H}(E)$ is obviously a weighted $\mathrm{PW}$-space. The converse statement however does not hold in general (Remark 3 on the last page of [1).

Lyubarskii and Seip made a bridge between Hermite-Biehler functions and a certain kind of potentials, namely, potentials of measures of the form $m(x) \mathrm{d} x$ for $m(x)$ measurable, positive, and $\simeq 1$. Such a potential cannot be defined as $\int_{-\infty}^{\infty} \log |1-z / t| m(t) \mathrm{d} t$. In fact, this last integral does not exist due to the dominating term in the expansion

$$
\log |1-z / t|=-x / t-\sum_{n=2}^{\infty}(1 / n) \Re\left(z^{n}\right) / t^{n}
$$

for $|t|$ large, where $z=x+\mathrm{i} y$. It suggests defining

$$
\omega_{m}(z)=\int_{-\infty}^{\infty} \log ^{*}|1-z / t| m(t) \mathrm{d} t
$$

where $\log ^{*}|1-z / t|=\log |1-z / t|+\chi(t) x / t, \quad \chi(t)=1-\chi_{[-1,1]}(t)$.

We first show that $\omega_{m}(z)$ is well-defined, indeed, that the above integral is absolutely convergent. The previous expansion gives, for $|t|$ large,

$$
\begin{aligned}
\left|\log ^{*}\right| 1-z / t|| & \leq \sum_{n=2}^{\infty}(1 / n)|z / t|^{n} \\
& \leq|z / t|^{2}(1 / 2-\log (1-|z / t|)) .
\end{aligned}
$$

Since $m(x) \simeq 1$, it suffices to show that $\int_{R}^{\infty}-\left(1 / t^{2}\right) \log (1-|z| / t) \mathrm{d} t<\infty$ for $R$ large. This last relation follows from the substitution $u=1-|z| / t$. Therefore, $\int_{-\infty}^{\infty}\left|\log ^{*}\right| 1-z / t|| \mathrm{d} t<\infty$. 
The inequality (2.3) and the dominated convergence theorem also yield that $\omega_{m}$ is continuous. The dominated convergence theorem then implies that for $z \notin \mathbb{R}$

$$
\partial_{y} \omega_{m}(z)=\pi P_{m}(z)
$$

where

$$
P_{m}(z)=\frac{1}{\pi} \int_{-\infty}^{\infty} \frac{y}{(x-t)^{2}+y^{2}} m(t) \mathrm{d} t
$$

is the Poisson transform of $m(x) \mathrm{d} x$. Similarly,

$$
\partial_{x} \omega_{m}(z)=\int_{-\infty}^{\infty}\left(\frac{x-t}{(x-t)^{2}+y^{2}}+\frac{\chi(t)}{t}\right) m(t) \mathrm{d} t
$$

for $z \notin \mathbb{R}$. Finally, a straight adaptation of the classical argument (Theorem 3.7.4 in [3]) gives $\Delta \omega_{m}=2 \pi m(x) \mathrm{d} x \mathrm{~d} \delta_{0}(y)$ in the sense of distribution, where $\delta_{0}$ denotes the 1-dimensional Dirac measure at 0 .

Example 2.2. For $m(x)=1$ and $z \notin \mathbb{R}, \partial_{y} \omega_{1}(z)=\pi \operatorname{sgn}(y)$, while $\partial_{x} \omega_{1}(z)=0$. Hence, $\omega_{1}(z)=\pi|y|+C$. By continuity, this last relation applies for all $z \in \mathbb{C}$. Since $\omega_{1}(0)=0$, we deduce $\omega_{1}(z)=\pi|y|$.

The aforementioned link between Hermite-Biehler functions and potentials of the form $\omega_{m}$ is given by the following multiplier lemma:

Proposition 2.3. Let $m(x) \simeq 1$ be a measurable function. There exists a HermiteBiehler function $E_{m}$ which satisfies

$$
\left|E_{m}(z)\right| \simeq \mathrm{e}^{\omega_{m}(z)} \text { when } \Im z \geq 0
$$

and whose zeroes are simple and of the form $\xi_{k}-\mathrm{i}$, where $\xi_{k+1}-\xi_{k} \simeq 1, \xi_{k} \in \mathbb{R}$.

Let $\varphi$ be the phase of $E_{m}$. By (2.2),

$$
\varphi^{\prime}(x)=\sum_{k} \frac{1}{\left(x-\xi_{k}\right)^{2}+1} .
$$

The condition $\xi_{k+1}-\xi_{k} \simeq 1$ then implies $\varphi^{\prime}(x) \simeq 1$. Therefore $\mathcal{H}\left(E_{m}\right)$ is a weighted $\mathrm{PW}$-space. By the multiplier lemma, $\left|E_{m}\right|$ may be replaced with $\mathrm{e}^{\omega_{m}}$ in the definition of $\mathcal{H}\left(E_{m}\right)$. Since $\omega_{m}(\bar{z})=\omega_{m}(z)$, it follows that $\mathcal{H}\left(E_{m}\right)$ is equal with equivalent norms to the space

$$
P W(m)=\left\{f \text { entire ; }\left\|f \mathrm{e}^{-\omega_{m}}\right\|_{2}<\infty,|f(z)| \mathrm{e}^{-\omega_{m}(z)}<C_{\varepsilon} \mathrm{e}^{\varepsilon|z|} \text { for } z \in \mathbb{C}\right\},
$$

equipped with the norm $\|f\|_{P W(m)}=\left\|f \mathrm{e}^{-\omega_{m}}\right\|_{2}$.

In particular, for any measurable $m(x) \simeq 1, P W(m)$ is a weighted $\mathrm{PW}$-space whose majorant-weight is comparable to $\left|E_{m}\right| \simeq \mathrm{e}^{\omega_{m}(x)}$. Consequently, given a realentire $g, \mathrm{e}^{g} P W(m)$ is also a weighted $\mathrm{PW}$-space; its majorant-weight is comparable to $\mathrm{e}^{g} \mathrm{e}^{\omega_{m}}$. The converse statement constitutes the remarkable achievement in [1]: Lyubarskii and Seip proved that all weighted $\mathrm{PW}$-spaces have a representation $\mathrm{e}^{g} P W(m)$, where $g$ is real-entire and $m \simeq 1$ is measurable.

Example 2.4. We have seen that $\omega_{1}(z)=\pi|y|$. Consequently, $P W(1)$ is the classical Paley-Wiener space, $L_{\pi}^{2}$. 


\section{Spaces Sharing a Given majorant-Weight}

In [1], Lyubarskii and Seip investigated the following question: which weighted $\mathrm{PW}$-spaces share a prescribed majorant-weight? They gave special attention to spaces of the form $\mathrm{e}^{a z} P W(m), a \in \mathbb{R}$, which we will call linearly reduced $\mathrm{PW}$ spaces. They obtained an answer for these last spaces, involving the following object: for $m$ measurable and $\simeq 1$ and for $\tau \in \mathbb{R}$,

$$
P W_{\tau}(m)=\left\{f \text { entire ; }\left\|f \mathrm{e}^{-\omega_{m}}\right\|_{2}<\infty,|f(z)| \mathrm{e}^{-\omega_{m}(z)} \leq C_{\varepsilon} \mathrm{e}^{\varepsilon|z|} \mathrm{e}^{\pi \tau|\Im z|}\right\} .
$$

Notice that $P W_{\tau}(m)=P W(m+\tau)$ if $m+\tau \simeq 1$, but this last relation is not assumed.

Proposition 3.1. Suppose $\mathrm{e}^{a_{0} x} \mathrm{e}^{\omega_{m_{0}}(x)} \simeq \mathrm{e}^{a x} \mathrm{e}^{\omega_{m}(x)}$ on the real axis, where $a, a_{0}$ are in $\mathbb{R}$ and $m, m_{0}$ are measurable and $\simeq 1$. Then, there exists a real number $b$ such that

$$
\mathrm{e}^{a_{0} x} \mathrm{e}^{\omega_{m_{0}}(z)} \simeq \mathrm{e}^{a x} \mathrm{e}^{\omega_{m}(z)} \mathrm{e}^{-\pi b|y|}
$$

on the whole complex plane, where $z=x+\mathrm{i} y$.

Proof. By hypothesis $\left|\omega_{m_{0}-m}(x)+\left(a_{0}-a\right) x\right|$ is bounded, and hence there exists a $C>0$ such that

$$
\begin{aligned}
\left|\omega_{m_{0}-m}(z)+\left(a_{0}-a\right) x\right| & \leq\left|\omega_{m_{0}-m}(z)-\omega_{m_{0}-m}(x)\right|+\left|\omega_{m_{0}-m}(x)+\left(a_{0}-a\right) x\right| \\
& \leq C(|y|+1) .
\end{aligned}
$$

In particular, $C y+\omega_{m_{0}-m}(z)+\left(a_{0}-a\right) x$ is bounded below on the upper half-plane and hence admits a Poisson representation

$$
D y+\frac{y}{\pi} \int_{-\infty}^{\infty} \frac{\omega_{m_{0}-m}(t)+\left(a_{0}-a\right) t}{(x-t)^{2}+y^{2}} \mathrm{~d} t \quad(y>0) .
$$

Observe that the last term in the previous expression is bounded. Consequently, letting $b=(C-D) / \pi$,

$$
\left|\pi b y+\omega_{m_{0}-m}(z)+\left(a_{0}-a\right) x\right| \lesssim 1 \quad(\Im z \geq 0)
$$

that is, $\mathrm{e}^{a_{0} x} \mathrm{e}^{\omega_{m_{0}}(z)} \simeq \mathrm{e}^{a x} \mathrm{e}^{\omega_{m}(z)} \mathrm{e}^{-b \pi \Im z}$ when $\Im z \geq 0$. The result follows.

Corollary 3.2. Each linearly reduced weighted $P W$-space whose majorant-weight is comparable to $\mathrm{e}^{a x} \mathrm{e}^{\omega_{m}(x)}$ is of the form $\mathrm{e}^{a z} P W_{-b}(m)$ for a certain $b \in \mathbb{R}$.

Remark 3.3. In their original paper Lyubarskii and Seip stated their result not in terms of linearly reduced PW-spaces, but in terms of linearly reduced majorantweights (that is, weights of the form $\mathrm{e}^{a x} \mathrm{e}^{\omega_{m}(x)}$ ). Their statement is too general: clearly, $\exp \left(\exp \left(-z^{2}\right)\right) P W(1)$ is a weighted $\mathrm{PW}$-space whose majorant-weight is $\simeq 1$, but it is not equal to $P W_{-b}(1)=L_{\pi(1-b)}^{2}$ for any $b$.

Linearly reduced PW-spaces of majorant $\mathrm{e}^{a x} \mathrm{e}^{\omega_{m}}(a \in \mathbb{R})$ thus take the form $\mathrm{e}^{a z} P W_{-b}(m)$, but for which possible $b$ ? Clearly each $b<\inf _{x \in \mathbb{R}} m(x)$ is possible, since then $P W_{-b}(m)=P W(m-b)$ with $m-b \simeq 1$. However, this majoration cannot be optimal in general, since it is easy to change the infimum of $m$ without

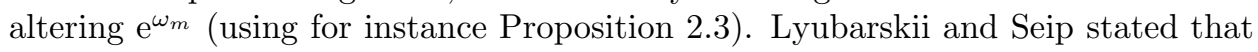
the optimal majoration is $b<D_{m}$, where

$$
D_{m}=\lim _{R \rightarrow \infty} \inf _{x \in \mathbb{R}} \frac{1}{2 R} \int_{-R}^{R} m(x+t) \mathrm{d} t
$$


is the uniform lower density of $m$. Notice that this last limit exists by Fekete's lemma, since $\inf _{x} \int_{-R}^{R} m(x+t) \mathrm{d} t=\inf _{x} \int_{0}^{2 R} m(x+t) \mathrm{d} t$ is superadditive.

3.1. The lower density is a majorant. For showing that $b<D_{m}$ is necessary, Lyubarskii and Seip argued by contradiction: they assumed that given an $\varepsilon>0$, for all sufficiently large $R$ there exists an $x_{R}$ satisfying

$$
\int_{-R}^{R}\left(m\left(x_{R}+s\right)-b\right) \mathrm{d} s \leq \varepsilon R .
$$

For convenience they set $x_{R}=0$. Let $\mathrm{e}^{a_{0} z} P W\left(m_{0}\right)$ be a representation of $\mathrm{e}^{a z} P W_{-b}(m)$ as a weighted Paley-Wiener space. By an elegant argument based on Green's formula they proved

$$
\left|\int_{-R}^{R}\left(m_{0}(t)-m(t)+b\right)\left(R^{2}-t\right) \mathrm{d} t\right| \lesssim R^{2} .
$$

They claimed however that (3.1) would imply

$$
2 \int_{0}^{R} t \int_{-t}^{t}\left(m_{0}(s)-m(s)+b\right) \mathrm{d} s \mathrm{~d} t \geq\left(\inf m_{0}\right) \frac{4}{3} R^{3}-\varepsilon R^{3},
$$

a contradiction. Unfortunately the use of the estimate

$$
2 \int_{0}^{R} t \int_{-t}^{t}(m(s)-b) \mathrm{d} s \mathrm{~d} t \leq \varepsilon R^{3}
$$

is not explicitly justified. A conscientious reader may get puzzled: one cannot for instance restrict the domain of integration to large $t$ and invoke (3.1) with $t$ instead of $R$, since $x_{t} \neq x_{R}$.

We prefer to present another proof, based on a new characterization of the uniform lower density:

Proposition 3.4. If $m \simeq 1$ is measurable, then

$$
D_{m}=\lim _{R \rightarrow \infty} \inf _{x \in \mathbb{R}} \frac{1}{R} \int_{0}^{R} \frac{1}{2 \rho} \int_{-\rho}^{\rho} m(x+t) \mathrm{d} t \mathrm{~d} \rho .
$$

Proof. For $R>0$ and $x \in \mathbb{R}$, let us write

$$
m_{R}(x)=\frac{1}{2 R} \int_{-R}^{R} m(x+t) \mathrm{d} t \text { and } A_{R}(x)=\frac{1}{R} \int_{0}^{R} m_{\rho}(x) \mathrm{d} \rho .
$$

In this notation we want to prove $D_{m}=\lim _{R \rightarrow \infty} \inf _{x} A_{R}(x)$, given that $D_{m}=$ $\lim _{R \rightarrow \infty} \inf _{x} m_{R}(x)$.

Given $\varepsilon>0$, let $R_{0}$ be such that $\left|\inf _{x} m_{\rho}(x)-D_{m}\right|<\varepsilon$ when $\rho \geq R_{0}$. Then, for $R$ large

$$
\inf _{x} A_{R}(x) \geq \frac{1}{R}\left(\int_{0}^{R_{0}}+\int_{R_{0}}^{R}\right) \inf _{x} m_{\rho}(x) \mathrm{d} \rho \geq \frac{R_{0} \inf m}{R}+\frac{R-R_{0}}{R}\left(D_{m}-\varepsilon\right) .
$$

Letting $R \rightarrow \infty$ along an appropriate sequence, we conclude

$$
\liminf _{R \rightarrow \infty} \inf _{x} A_{R}(x) \geq D_{m} .
$$


Let us derive the converse inequality. Let $0<\varepsilon<1 / 4$ be given, and define $\eta=\varepsilon / \log (1 / 2 \varepsilon)$. There exists an $R_{0}$ depending on $\varepsilon$ such that

$$
\left|\inf _{x} m_{\rho}(x)-D_{m}\right|<\eta \quad \text { whenever } \rho \geq R_{0} .
$$

Let $R \geq R_{0}$ be arbitrarily fixed, and let $S=R / \varepsilon$. By the previous relation there exists an $x^{*}$ such that $m_{S}\left(x^{*}\right)<D_{m}+2 \eta$. Consequently, for $\rho<S-2 R$

$$
\frac{S-\rho}{2 S} m_{\frac{S-\rho}{2}}\left(x^{*}-\frac{S+\rho}{2}\right)+\frac{\rho}{S} m_{\rho}\left(x^{*}\right)+\frac{S-\rho}{2 S} m_{\frac{S-\rho}{2}}\left(x^{*}+\frac{S+\rho}{2}\right)<D_{m}+2 \eta
$$

(since the left-hand side is equal to $m_{S}\left(x^{*}\right)$ ), while

$$
m_{\frac{S-\rho}{2}}\left(x^{*} \pm \frac{S+\rho}{2}\right) \geq \inf _{x} m_{\frac{S-\rho}{2}}(x)>D_{m}-\eta .
$$

It follows that $m_{\rho}\left(x^{*}\right)<D_{m}+\frac{3 \eta S}{\rho}$ for such $\rho$. Therefore, for $\rho \in[R, S-2 R]$

$$
A_{\rho}\left(x^{*}\right)=\frac{1}{\rho}\left(\int_{0}^{R}+\int_{R}^{\rho}\right) m_{r}\left(x^{*}\right) \mathrm{d} r \leq \frac{R \sup m}{\rho}+\frac{3 \eta S}{\rho} \log \left(\frac{\rho}{R}\right)+D_{m} .
$$

Letting $\rho=S / 2=R / 2 \varepsilon$, the definition of $\eta$ yields

$$
\inf _{x \in \mathbb{R}} A_{R / 2 \varepsilon}(x) \leq A_{R / 2 \varepsilon}\left(x^{*}\right) \leq D_{m}+C \varepsilon,
$$

where $C=2 \sup m+6$. This last relation holds for all $R \geq R_{0}$. In other words

$$
\sup _{R \geq R_{0} / 2 \varepsilon} \inf _{x \in \mathbb{R}} A_{R}(x) \leq D_{m}+C \varepsilon .
$$

Therefore, $\lim \sup _{R \rightarrow \infty} \inf _{x} A_{R}(x) \leq D_{m}+C \varepsilon$. Since $\varepsilon>0$ is arbitrarily small, we conclude

$$
\limsup _{R \rightarrow \infty} \inf _{x \in \mathbb{R}} A_{R}(x) \leq D_{m}
$$

as desired.

The following identity is also useful:

Lemma 3.5. For $m$ measurable and bounded and $R>0$,

$$
\frac{1}{\pi} \int_{0}^{\pi} \omega_{m}\left(R \mathrm{e}^{\mathrm{i} \theta}\right) \mathrm{d} \theta=\int_{0}^{R} \frac{1}{\rho} \int_{-\rho}^{\rho} m(t) \mathrm{d} t \mathrm{~d} \rho .
$$

Proof. Fubini's theorem and the relation $\int_{0}^{\pi} \cos \theta \mathrm{d} \theta=0$ give

$$
\frac{1}{\pi} \int_{0}^{\pi} \omega_{m}\left(R \mathrm{e}^{\mathrm{i} \theta}\right) \mathrm{d} \theta=\frac{1}{\pi} \int_{-\infty}^{\infty} m(t) \int_{0}^{\pi} \log \left|1-\frac{R \mathrm{e}^{\mathrm{i} \theta}}{t}\right| \mathrm{d} \theta \mathrm{d} t
$$

Moreover, Jensen's formula gives

$$
\frac{1}{\pi} \int_{0}^{\pi} \log \left|1-\frac{R \mathrm{e}^{\mathrm{i} \theta}}{t}\right| \mathrm{d} \theta=\frac{1}{2 \pi} \int_{-\pi}^{\pi} \log \left|1-\frac{R \mathrm{e}^{\mathrm{i} \theta}}{t}\right| \mathrm{d} \theta=\chi_{[-R, R]}(t) \log (R /|t|) .
$$

Therefore,

$$
\frac{1}{\pi} \int_{0}^{\pi} \omega_{m}\left(R \mathrm{e}^{\mathrm{i} \theta}\right) \mathrm{d} \theta=\int_{-R}^{R} \log (R /|t|) m(t) \mathrm{d} t=\int_{0}^{R} \frac{1}{\rho} \int_{-\rho}^{\rho} m(t) \mathrm{d} t \mathrm{~d} \rho,
$$

by Fubini's theorem again.

Proposition 3.6. In Corollary 3.2, $b<D_{m}$. 
Proof. Let $\mathrm{e}^{a_{0} z} P W\left(m_{0}\right)=\mathrm{e}^{a z} P W_{-b}(m)$ be the linearly reduced space in question, and suppose by contradiction that $b \geq D_{m}$. By our characterization of $D_{m}$, for any large $R$ there exists an $x_{R}$ such that

$$
\frac{1}{R} \int_{0}^{R} \frac{1}{2 \rho} \int_{-\rho}^{\rho}\left(m\left(x_{R}+t\right)-b\right) \mathrm{d} t \mathrm{~d} \rho \leq \frac{\inf m_{0}}{2} .
$$

In particular, for $R$ large

$$
\frac{1}{R} \int_{0}^{R} \frac{1}{2 \rho} \int_{-\rho}^{\rho}\left(m_{0}\left(x_{R}+t\right)-m\left(x_{R}+t\right)+b\right) \mathrm{d} t \mathrm{~d} \rho \geq \frac{\inf m_{0}}{2} .
$$

The last lemma then implies

$$
\frac{1}{2 \pi R} \int_{0}^{\pi}\left(\omega_{m_{0}\left(x_{R}+\cdot\right)}\left(R \mathrm{e}^{\mathrm{i} \theta}\right)-\omega_{m\left(x_{R}+\cdot\right)}\left(R \mathrm{e}^{\mathrm{i} \theta}\right)+\pi b R \sin \theta\right) \mathrm{d} \theta \geq \frac{\inf m_{0}}{2} .
$$

Notice that in general

$$
\omega_{M(X+\cdot)}(z)=\omega_{M}(z+X)-\omega_{M}(X)+\Re z \int_{-\infty}^{\infty}\left(\frac{\chi(t-X)}{t-X}-\frac{\chi(t)}{t}\right) M(t) \mathrm{d} t,
$$

and hence

$$
\int_{0}^{\pi} \omega_{M(X+\cdot)}\left(R \mathrm{e}^{\mathrm{i} \theta}\right) \mathrm{d} \theta=\int_{0}^{\pi}\left(\omega_{M}\left(R \mathrm{e}^{\mathrm{i} \theta}+X\right)-\omega_{M}(X)\right) \mathrm{d} \theta .
$$

Therefore,

$$
\frac{1}{2 \pi R} \int_{0}^{\pi}\left(\omega_{m_{0}-m}\left(R \mathrm{e}^{\mathrm{i} \theta}+x_{R}\right)+\pi b R \sin \theta-\omega_{m_{0}-m}\left(x_{R}\right)\right) \mathrm{d} \theta \geq \frac{\inf m_{0}}{2} .
$$

However, Proposition 3.1 implies that

$$
\left|\omega_{m_{0}-m}\left(R \mathrm{e}^{\mathrm{i} \theta}+x_{R}\right)+\pi b R \sin \theta+\left(a_{0}-a\right)\left(R \cos \theta+x_{R}\right)\right| \lesssim 1,
$$

while $\left|\omega_{m_{0}-m}\left(x_{R}\right)+\left(a_{0}-a\right) x_{R}\right| \lesssim 1$. Since $\int_{0}^{\pi} R \cos \theta \mathrm{d} \theta=0$, the integral in the relation (3.2) is bounded, a contradiction.

3.2. The lower density is the least majorant. For showing that any $b<D_{m}$ is suitable, Lyubarskii and Seip replaced $m$ with a smoothing of $m$ of the form

$$
m_{R}(x)=\frac{1}{2 R} \int_{-R}^{R} m(x+t) \mathrm{d} t .
$$

They justified this replacement by the relation $\left|\omega_{m}(z)-\omega_{m_{R}}(z)\right| \lesssim 1$, which is essentially right (after addition of a linear term $\alpha x$ ). In fact, $\omega_{m_{R}}(z)-\omega_{m}(z)$ is equal to

$$
\begin{aligned}
& \frac{1}{2 R} \int_{-R}^{R}\left(\int_{-\infty}^{\infty} \log ^{*}|1-z / t| m(t+s) \mathrm{d} t-\int_{-\infty}^{\infty} \log ^{*}|1-z / t| m(t) \mathrm{d} t\right) \mathrm{d} s \\
& =\frac{1}{2 R} \int_{-R}^{R} \int_{-\infty}^{\infty}\left(\log \left|1-\frac{z}{t-s}\right|+\frac{\chi(t-s)}{t-s} x-\log \left|1-\frac{z}{t}\right|-\frac{\chi(t)}{t} x\right) m(t) \mathrm{d} t \mathrm{~d} s \\
& =F(z)+\alpha x,
\end{aligned}
$$


where

$$
\begin{gathered}
F(z)=\frac{1}{2 R} \int_{-R}^{R} \int_{-\infty}^{\infty} \log \frac{|1-z /(t-s)|}{|1-z / t|} m(t) \mathrm{d} t \mathrm{~d} s \quad \text { and } \\
\alpha=\frac{1}{2 R} \int_{-R}^{R} \int_{-\infty}^{\infty}\left(\frac{\chi(t-s)}{t-s}-\frac{\chi(t)}{t}\right) m(t) \mathrm{d} t \mathrm{~d} s .
\end{gathered}
$$

Notice that both $\alpha$ and $\omega_{m_{R}-m}(z)$ are absolutely convergent, forcing $F(z)$ to be such.

Let us prove that $F(z)$ is bounded. In fact, $\int_{-\infty}^{\infty} \log \frac{|1-x /(t-s)|}{|1-x / t|} m(t) \mathrm{d} t$ is equal to

$$
\omega_{m(x+\cdot)}(s)-\omega_{m}(s)+s \int_{-\infty}^{\infty}\left(\frac{\chi(t)}{t}-\frac{\chi(t-x)}{t-x}\right) m(t) \mathrm{d} t .
$$

Clearly $\left|\omega_{m(x+\cdot)}(s)\right| \lesssim \int_{-\infty}^{\infty}\left|\log ^{*}\right| 1-(s / t)|| \mathrm{d} t<\infty$ uniformly in $s \in[-R, R]$, and similarly for $\left|\omega_{m}(s)\right|$. Moreover, the last term in (3.3) disappears when averaging over $s \in[-R, R]$. Hence, $|F(z)| \lesssim 1$.

In total, $\mathrm{e}^{\omega_{m}(z)}$ is comparable to $\mathrm{e}^{\omega_{m_{R}}}(z) \mathrm{e}^{-\alpha x}$, and hence $P W_{-b}(m)$ equals $\mathrm{e}^{-\alpha z} P W\left(m_{R}-b\right)$, which is a weighted PW-space for all $b<\inf _{x} m_{R}(x)$, eventually for all $b<D_{m}$.

Joining this result with Corollary 3.2 and Proposition 3.6, we have completed the proof of the following theorem:

Theorem 3.7. Let $m \simeq 1$ be measurable and $a \in \mathbb{R}$. The family of linearly reduced weighted Paley-Wiener spaces whose majorant-weight is comparable to $\mathrm{e}^{a x} \mathrm{e}^{\omega_{m}(x)}$ consists of all $\mathrm{e}^{a z} P W_{-b}(m)$ with $b<D_{m}$.

\section{ACKNOWLEDGEMENTS}

The author would like to thank Professor Kristian Seip for his constant support over the years, his valuable comments, and his conscientious reading. His kind visit at UAEU has been the source of stimulating discussions among colleagues who believe in the beauty of mathematics. The author would also like to thank his collaborator, Professor Yurii Lyubarskii, who accepted with Professor Seip to co-supervise his postdoctoral fellowship at NTNU a few years ago.

The author also thanks Professor Marco Merkli and his wife, Professor Nancy Pedri, for their warm welcome at Memorial University in the summer of 2010. Thanks to them, the author could benefit from valuable resources for doing research, in addition to enjoying their hospitality, and wishes them a great continuation.

\section{REFERENCES}

[1] Y. Lyubarskii and K. Seip, Weighted Paley-Wiener spaces, J. Amer. Math. Soc. 5 (2002), 979-1006. MR.1915824(2003m:46039)

[2] L. de Branges, Hilbert Spaces of Entire Functions, Prentice-Hall, Englewood Cliffs, 1968. MR0229011 (37:4590)

[3] T. Ransford, Potential Theory in the Complex Plane, London Math. Soc. Student Texts 28, Cambridge University Press, 1995. MR1334766 (96e:31001)

Department of Mathematical Sciences, United Arab Emirates University, P.O. Box 17551, Al Ain, Abu Dhabi, United Arab Emirates

E-mail address: PhilippePoulin@uaeu.ac.ae 